09,12

\title{
К расчету оптических характеристик и размерных сдвигов поверхностных плазмонов сферических биметаллических наночастиц
}

\author{
(C) A.В. Коротун, В.В. Погосов \\ Национальный университет „Запорожская политехника“, \\ Запорожье, Украина \\ E-mail: andko@zntu.edu.ua, vpogosov@zntu.edu.ua
}

Поступила в Редакцию 6 сентября 2020 г.

В окончательной редакции 6 сентября 2020 г.

Принята к публикации 8 сентября 2020 г.

\begin{abstract}
Получены формулы для эффективного времени релаксации, когда длина свободного пробега электронов меньше или сравнима с характерными размерами металлических областей. Рассчитана частотная дисперсия оптических характеристик сферических биметаллических частиц вблизи плазменных резонансов в отсутствии квантово-размерных эффектов. Сохраняя стиль общепринятого описания монометаллических частиц на основе теорий Друде и Ми, проанализирована частотная зависимость электрической дипольной поляризуемости двухслойной металлической наносферы. Появление двух максимумов поляризуемости является следствием различия металлов ядра и оболочки. Расчеты были проведены для частиц $\mathrm{Au} @ \mathrm{Ag}, \mathrm{Ag} @ \mathrm{Au}$, $\mathrm{Au} @ \mathrm{Pt}, \mathrm{Pt} @ \mathrm{Au}$ и Pt@Pd, погруженных в тефлон. Продемонстрована возможность управления оптическими характеристиками биметаллических частиц путем изменения их состава и объемного содержания металлов. Проведены расчеты сечений поглощения и рассеяния, а также оптической радиационной эффективности частиц в широком спектральном диапазоне. Оценена возможная температура биметаллических частиц при поглощении электромагнитной волны (для целей фототермической терапии злокачественных опухолей).
\end{abstract}

Ключевые слова: биметаллическая наночастица, поверхностный плазмон, поляризуемость, сечение поглощения, сечение рассеяния, время релаксации.

DOI: 10.21883/FTT.2021.01.50409.178

\section{1. Введение}

В последние десятилетия интенсивно исследуются композитные материалы на основе металлических наночастиц [1-4]. Это связано с тем, что в поле электромагнитной волны на наночастице локализируется оптическая энергия вследствие возбуждения поверхностных плазмонов. При этом интенсивность падающего излучения вблизи поверхности наночастицы может возрастать на несколько порядков. В связи с этим, металлические наночастицы широко применяются в плазмонной нанолитографии [5], оптической микроскопии ближнего поля [6], астигматическом оптическом пинцете [7], для усиления поля рамановского рассеяния [8], сенсорных и фотогальванических устройствах $[9,10]$, плазмонной фотовольтаике [11], фотокатализе [12] и терапии злокачественных опухолей [13]. В качестве плазмонных материалов чаще всего используют $\mathrm{Au}$ и $\mathrm{Ag}$, поскольку для них резонансная частота локализованных поверхностных плазмонов лежит в видимой области спектра, а сами наночастицы химически стабильны и просты в приготовлении [14]. Однако, фотофункциональные металлы, такие как $\mathrm{Pd}$ и Pt, могут быть использованы в качестве фотокатализаторов [15]. Кроме того, эффективность фотокаталитической активности диоксида титана $\left(\mathrm{TiO}_{2}\right)$, который используется для очистки окружающей среды, может быть увеличена путем осаждения частиц Pt. Такие частицы соответствующих размеров позволяют осуществить эффективное разделение электронов и дырок, что приводит к усилению фотокаталитической активности [16].

Металлические наночастицы можно использовать для повышения эффективности работы полупроводниковых солнечных элементов [11]. Наличие металлических наночастиц приводит к рассеянию падающего солнечного света вглубь полупроводника, благодаря чему возрастает доля поглощенного полупроводником света. Это позволяет уменьшить физическую толщину фотовольтаического элемента при сохранении его оптической толщины. Существует определенный оптимальный диапазон размеров металлических частиц, поскольку очень малые частицы сильно поглощают свет, что приводит к потерям. В то же время их размер должен соответствовать условиям возбуждения локализованного поверхностного резонанса, т. е. не должен превышать длину волны света. Наряду с элементным составом, свойства поверхностного плазмонного резонанса существенно зависят от размера, формы и внутренней структуры наночастиц [2]. Так, например, пик поверхностного плазмонного резонанса при деформации сферической наночастицы Au в цилиндрическую расщепляется на два - поперечный и продольный. Таким образом, число резонансных мод и положение пика поверхностного плазмонного резонанса 
можно настраивать, манипулируя любым из указанных параметров или их комбинациями.

По сравнению с монометаллическими наночастицами биметаллические частицы более эффективны в регулировании плазмонного резонанса [17-20]. Состав биметаллических наночастиц можно изменять с целью управления свойствами плазмонов, поскольку для разных металлов они располагаются в разных областях спектра. Кроме того, положение и профиль резонанса локализованных поверхностных плазмонов, а также их количество сильно зависит от морфологии биметаллической частицы, т. е. пространственного расположения и упорядочения двух различных типов атомов [21]. Например, в сплавной частице $\mathrm{Ag}-\mathrm{Au}$ положение пика плазмонного резонанса отличается от первого по частоте пика в частице типа „ядро-оболочка“ $(\mathrm{Au} @ \mathrm{Ag})$, несмотря на одинаковое молярное соотношение $\mathrm{Ag} / \mathrm{Au}$ [22].

Переходные металлы, такие как $\mathrm{Pd}$, также используются в фототермической терапии злокачественных новообразований. Этот материал, соединяясь, например, с наноструктурами $\mathrm{Au}$, которые являются наилучшим вариантом в фототермической терапии, смещает в низкочастотную область и расширяет спектр поглощения по сравнению с монометаллическими наночастицами $\mathrm{Au}$. Это явление максимизирует поглощение в ближней инфракрасной области и, следовательно, повышает фототермический отклик структур на лазерное облучение. В дополнение к многочисленным свойствам и применению $\mathrm{Pd}$ и $\mathrm{Pt}$, существуют значительные различия между диэлектрическими постоянными этих переходных металлов и благородных металлов, таких как $\mathrm{Au}$ и $\mathrm{Ag}$ [14]. Тем не менее интерпретация плазмонных резонансов, основанная на модели свободных электронов, хорошо применимая к таким металлам, как $\mathrm{Ag}$ и $\mathrm{Au}$, может быть распространена на Pd и Pt. Таким образом, исследование и сравнение оптических откликов наночастиц $\mathrm{Pd} @ \mathrm{Pt}$ и наночастиц других металлов представляет большой интерес.

Поэтому, в связи с широкими перспективами использования биметаллических частиц, исследование их оптических характеристик, а также демонстрация возможности управления их свойствами путем изменения состава и геометрических параметров ядра и оболочки, является актуальной задачей.

Целью настоящей работы является расчет частотной дисперсии оптических характеристик сферических биметаллических частиц вблизи плазменных резонансов в отсутствии квантово-размерных эффектов. При этом сохраняется стиль общепринятого описания монометаллических частиц на основе теорий Друде и Ми.

\section{2. Теория}

Как известно, особенности взаимодействия электромагнитных волн с металлическими частичками отражаются в частотной дисперсии дипольной поляризуемости.

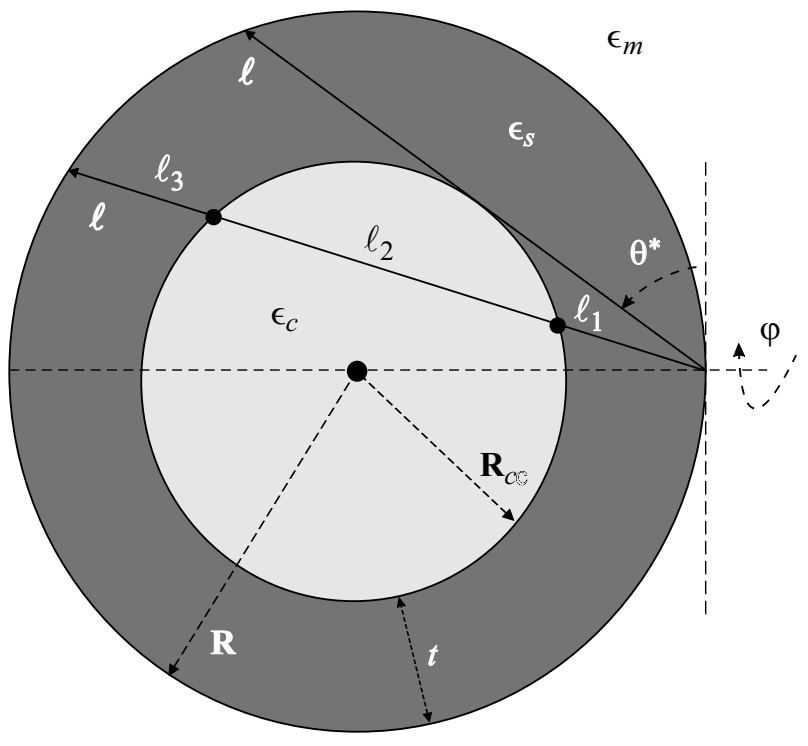

Рис. 1. Биметаллическая наночастица и ее геометрические параметры (классические траектории электронов комментируются в Приложении).

Рассмотрим биметаллическую сферу общего радиуса $R$ в диэлектрической матрице с постоянной $\epsilon_{\mathrm{m}}$. Ядро радиусом $R_{\mathrm{c}}$ покрыто оболочкой толщиной $t, R_{\mathrm{c}}+t=R$ (рис. 1). Как следует из электродинамики [23], дипольная поляризуемость $\alpha_{@}$ такой частицы може быть записана в виде

$$
\frac{\alpha_{@}}{R^{3}}=\frac{\left(\epsilon_{\mathrm{s}}-\epsilon_{\mathrm{m}}\right)\left(2 \epsilon_{\mathrm{s}}+\epsilon_{\mathrm{c}}\right)-\left(2 \epsilon_{\mathrm{s}}+\epsilon_{\mathrm{m}}\right)\left(\epsilon_{\mathrm{s}}-\epsilon_{\mathrm{c}}\right) \beta_{\mathrm{c}}}{\left(\epsilon_{\mathrm{s}}+2 \epsilon_{\mathrm{m}}\right)\left(2 \epsilon_{\mathrm{s}}+\epsilon_{\mathrm{c}}\right)-\left(2 \epsilon_{\mathrm{s}}-\epsilon_{\mathrm{m}}\right)\left(\epsilon_{\mathrm{s}}-\epsilon_{\mathrm{c}}\right) \beta_{\mathrm{c}}},
$$

где величины $\alpha_{@}, \epsilon_{\mathrm{s}}, \epsilon_{\mathrm{c}}$ являются функциями частоты света $\omega, \beta_{\mathrm{c}}=R_{\mathrm{c}}^{3} / R^{3}$ - объемная „фракция“ материала ядра частицы,

$$
0 \leq \beta_{\mathrm{c}}<1 \text {. }
$$

Если $R_{\mathrm{c}}$ или $t$ равны нулю $\left(\beta_{\mathrm{c}}=0\right.$ или 1$)$, то биметаллическая частица становится монометаллической $\left(\epsilon_{\mathrm{s}}=\epsilon_{\mathrm{c}} \equiv \epsilon\right)$. Однако формула (1) выведена при условии ненулевой толщины оболочки $\left(\beta_{\mathrm{c}}<1\right)$, т.е. с учетом условий на границе раздела оболочки и диэлектрика матрицы. Использовать равенство $\beta_{\mathrm{c}}=1$ следует с осторожностью, поскольку это может привести к нефизическим результатам при описании плазмонного резонанса (см. комментарий к формуле (24)).

Запишем выражение (1) в более компактном виде

$$
\frac{\alpha_{@}}{R^{3}}=\frac{\epsilon_{@}-\epsilon_{\mathrm{m}}}{\epsilon_{@}+2 \epsilon_{\mathrm{m}}},
$$

вводя диэлектрическую функцию двухслойной сферы

$$
\epsilon_{@}=\epsilon_{\mathrm{s}} \frac{1+2 \beta_{\mathrm{c}} \delta_{@}}{1-\beta_{\mathrm{c}} \delta_{@}}, \quad \delta_{@} \equiv \frac{\epsilon_{\mathrm{c}}-\epsilon_{\mathrm{s}}}{\epsilon_{\mathrm{c}}+2 \epsilon_{\mathrm{s}}} .
$$

Далее необходимо выбрать рабочее выражение для диэлектрических функций. 
Простой и удобной для анализа является популярное модифицированное выражение теории Друде-ЛоренцаЗоммерфельда, которое мы применяем к описанию электронов ядра (оболочки) в отсутствии квантоворазмерного эффекта

$$
\epsilon_{\mathrm{c}(\mathrm{s})}=\epsilon_{\mathrm{c}(\mathrm{s})}^{\infty}-\frac{\omega_{b p, \mathrm{c}(\mathrm{s})}^{2}\left(\tau_{\mathrm{c}(\mathrm{s})}^{\mathrm{eff}}\right)^{2}}{1+\left(\omega \tau_{\mathrm{c}(\mathrm{s})}^{\mathrm{eff}}\right)^{2}}+\mathrm{i} \frac{\omega_{b p, \mathrm{c}(\mathrm{s})}^{2} \tau_{\mathrm{c}(\mathrm{s})}^{\mathrm{eff}}}{\omega\left[1+\left(\omega \tau_{\mathrm{c}(\mathrm{s})}^{\mathrm{eff}}\right)^{2}\right]},
$$

где константа $\epsilon_{\mathrm{c}(\mathrm{s})}^{\infty}$ соответствует вкладу ионного остова металла ядра или оболочки, соответственно

$$
\omega_{b p, \mathrm{c}(\mathrm{s})}^{2}=\frac{\bar{n}_{\mathrm{c}(\mathrm{s})} e^{2}}{\epsilon_{0} m_{\mathrm{c}(\mathrm{s})}^{\mathrm{eff}}}
$$

- объемная плазменная частота, $\epsilon_{0}$ - диэлектрическая постоянная, $\bar{n}, m^{\mathrm{eff}}$ и $\tau^{\mathrm{eff}}-$ концентрация, эффективные масса и время релаксации электронов, соответственно; $\bar{n}=3 /\left(4 \pi r_{s}^{3}\right), r_{s}-$ среднее расстояние между электронами.

Плазмонные возбуждения могут затухать безизлучательно, передавая энергию решетке, или излучательно. Начиная с больших частиц, при уменьшении их размеров, кроме рассеяния электронов на фононах, примесях и других дефектах, необходимо учитывать радиационное излучение электронов, а в случае, когда характерные размеры становятся меньше длины свободного пробега электронов в объеме, необходимо учитывать также рассеяние на поверхности частицы.

Для монометаллических сфер эффективное время релаксации $\tau^{\text {eff }}$ определяется выражением

$$
\frac{1}{\tau^{\text {eff }}}=\frac{1}{\tau^{\text {bulk }}}+\frac{1}{\tau^{\text {surf }}}+\frac{1}{\tau^{\text {rad }}},
$$

где $\tau^{\text {bulk }}$ - время релаксации в объеме 3D-металла.

Второе слагаемое в выражении (5), обусловленное рассеянием электронов на поверхности, в литературе принято записывать в виде [24]:

$$
\frac{1}{\tau^{\text {surf }}}=\frac{v_{\mathrm{F}}}{\langle\ell\rangle_{R}} \equiv \mathscr{A} \frac{v_{\mathrm{F}}}{R},
$$

где $\langle\ell\rangle_{R}-$ средняя длина свободного пробега,

$$
v_{\mathrm{F}}=\frac{\hbar}{m^{\mathrm{eff}}}\left(3 \pi^{2} \bar{n}\right)^{1 / 3}
$$

\section{- скорость фермиевских электронов.}

Коэффициент $\mathcal{A}$ в (6) - эффективный параметр, описывающий степень потери когерентности при рассеянии электрона на поверхности частицы. Так, для классического (диффузного) режима рассеяния $\mathcal{A}=1$ [24], а для изотропного $-\mathcal{A}=3 / 4$. В общем случае параметр $\mathcal{A}$ может быть функцией частоты падающего света и латерального размера системы [25,26] (см., например, рис. 4 в [26], где сравнивается рассчитанная зависимость $\mathcal{A}(\omega, R)$ для наночастиц $\mathrm{Ag}$ с экспериментами).
Последний из учитываемых факторов уширения плазмонных линий в (5) это радиационный вклад, обусловленный излучением ускоренных внутренним электрическим полем электронов во всех направлениях [27],

$$
\frac{1}{\tau^{\mathrm{rad}}}=\frac{2}{9} \frac{N e^{2} \omega}{m^{\mathrm{eff}} c^{3} \epsilon_{\mathrm{m}}^{1 / 2} \epsilon_{0}^{2}} \operatorname{Re} \sigma(\omega),
$$

где $N$ - полное число электронов проводимости, $\operatorname{Re} \sigma(\omega)$ - действительная часть проводимости металла.

Условием равноправного учета $\tau^{\text {surf }}$ и $\tau^{\text {rad }}$ для монометаллических частиц является неравенство $\ell^{\text {bulk }}>2 R$ для длины свободного пробега электронов в объеме ( $\ell^{\text {bulk }}$ оценивается как $\left.v_{\mathrm{F}} \tau^{\text {bulk }}\right)$. При выполнении условия $\ell^{\text {bulk }}<2 R$ роль радиационного затухания становится превалирующей [27].

В случае биметаллических частиц аналогичные неравенства

$$
\begin{array}{ll}
\ell_{\mathrm{c}}^{\text {bulk }}>2 R_{\mathrm{c}}, & \ell_{\mathrm{s}}^{\text {bulk }}>2 t, \\
\ell_{\mathrm{c}}^{\text {bulk }} \leq 2 R_{\mathrm{c}}, & \ell_{\mathrm{s}}^{\text {bulk }} \leq 2 t
\end{array}
$$

мы будем распространять на металлические области ядра и оболочки.

Как оказалось, при сохранении стиля вычисления частот столкновения (5)-(8) для составной металлической частицы, возникли принципиальные трудности. Если для вычисления $\tau$ surf достаточно было найти среднюю длину свободного пробега $\langle\ell\rangle_{R}$, то для мультиметаллической частицы следует уже рассчитывать среднее время $\left\langle\tau_{@}^{\text {surf }}\right\rangle_{R}$. Это является следствием того, что некоторые траектории электронов пересекают области разных металлов (рис. 1). Такое же замечание относится и к остальным компонентам эффективной частоты столкновения. Теперь и для них необходима четкость в определении траектории с учетом параметров металлов, через которые они проходят. Четкость траектории подразумевает способность выразить ее через геометрические параметры частицы. А это можно сделать только тогда, когда начало и конец классической траектории находится на поверхности. Таким образом все времена в

$$
\frac{1}{\tau_{@}^{\text {eff }}}=\frac{1}{\left\langle\tau_{@}^{\text {bulk }}\right\rangle_{R}}+\frac{1}{\left\langle\tau_{@}^{\text {surf }}\right\rangle_{R}}+\frac{1}{\left\langle\tau_{@}^{\mathrm{rad}}\right\rangle_{R}}
$$

записываются в виде усредненных величин (их вывод приведен в Приложении).

Слагаемые в (11) являются своего рода „перекрестными“ и создают эффект „внутреннего перемешивания“ диэлектрических функций $\epsilon_{\mathrm{c}}$ и $\epsilon_{\mathrm{s}}$ в нашей задаче, как, например, это происходит при „внешнем перемешивании“ диэлектрических функций согласно формуле (1). Их нельзя отнести целиком либо к ядру либо к оболочке. Вследствие этого, в используемые при прямых вычислениях выражения (4) для ядра и оболочки, необходимо заменить величины $\tau_{\mathrm{c}(\mathrm{s})}^{\mathrm{eff}}$ на выражение $\tau_{@}^{\text {eff }}$ из $(11)$. 
Зная поляризуемость, можно определить сечения поглощения $C^{\mathrm{abs}}$ и рассеяния $C^{\mathrm{sca}}$ световой волны частицей. Эти величины представляют наибольший интерес, поскольку являются экспериментально измеряемыми. Так, сечение рассеяния измеряется в темнопольных экспериментах, а сечение экстинкции $C^{\mathrm{ext}}=C^{\mathrm{abs}}+C^{\mathrm{sca}}-$ в измерениях пропускания.

Ограничиваясь дипольным приближением, сечения поглощения $C_{@}^{\mathrm{abs}}$ и рассеяния $C_{@}^{\mathrm{sca}}$ для биметаллических сфер определяются электрической $\alpha_{@}$ и магнитной $\alpha_{@}^{\mathrm{mag}}$ поляризуемостями $[1,28]$ :

$$
\begin{gathered}
C_{@}^{\mathrm{abs}}=\frac{4 \pi \omega}{c} \epsilon_{\mathrm{m}}^{1 / 2} \operatorname{Im}\left(\alpha_{@}+\alpha_{@}^{\mathrm{mag}}\right), \\
C_{@}^{\mathrm{sca}}=\frac{\omega^{4} \epsilon_{\mathrm{m}}^{2}}{6 \pi c^{4}}\left|\alpha_{@}+\alpha_{@}^{\mathrm{mag}}\right|^{2},
\end{gathered}
$$

где $c-$ скорость света, а при условии однородности поля электромагнитной волны в частице [29]

$$
\xi=\frac{\omega R}{c}\left|\epsilon_{\mathrm{m}} \epsilon_{@}(\omega)\right|^{1 / 2} \ll 1,
$$

выражение для магнитной поляризуемости имеет вид

$$
\alpha_{@}^{\mathrm{mag}}=\left(\frac{\omega R}{c}\right)^{2} \epsilon_{\mathrm{m}} \frac{R^{3}}{30}\left(\epsilon_{@}-\epsilon_{\mathrm{m}}\right) .
$$

В инфракрасной области спектра с увеличением радиуса $R$ монометаллической сферы вклад $\operatorname{Im} \alpha^{\mathrm{mag}}$ в (12)-(15) возрастает и при $R \approx 2 \mathrm{~nm}$ величины $\operatorname{Im} \alpha$ и $\operatorname{Im} \alpha^{\mathrm{mag}}$ становятся примерно одинаковыми [28]. В нашей задаче мы будем пренебрегать $\operatorname{Im} \alpha^{\mathrm{mag}}$, вследствие того, что вблизи плазмонного резонанса $\epsilon_{@}(\omega) \rightarrow 0$ и $\operatorname{Im} \alpha^{\mathrm{mag}} \ll \operatorname{Im} \alpha$.

В качестве характеристики „рассеивающей способности“ наночастиц используется оптическая радиационная эфбективность, величина которой рассчитывается как [30]:

$$
\xi_{@}^{\mathrm{rad}}=\frac{1}{1+C_{@}^{\mathrm{abs}} / C_{@}^{\mathrm{sca}}} .
$$

Величина $\xi_{@}^{\mathrm{rad}}$ характеризует отношение энергии электромагнитного поля, рассеиваемой наночастицами, к энергии падающей электромагнитной волны и считается важной для эффективной работы полупроводниковых солнечных батарей, содержащих эти частицы.

\section{3. Результаты вычислений и их обсуждение}

Расчеты частотных зависимостей поляризуемости, сечений поглощения и рассеяния, а также оптической радиационной эффективности были проведены для наносфер Au@Ag, Ag@Au, Au@Pt, Pt@Au и Pt@Pd, pacположенных в тефлоне $\left(\epsilon_{\mathrm{m}}=2.3\right)$. Параметры металлов приведены в таблице.

С целью определения влияния компонент эффективного времени релаксации на рис. 2 приведена частотная зависимость $\operatorname{Im} \alpha_{@}$ составной частицы $\mathrm{Au} @ \mathrm{Ag}$

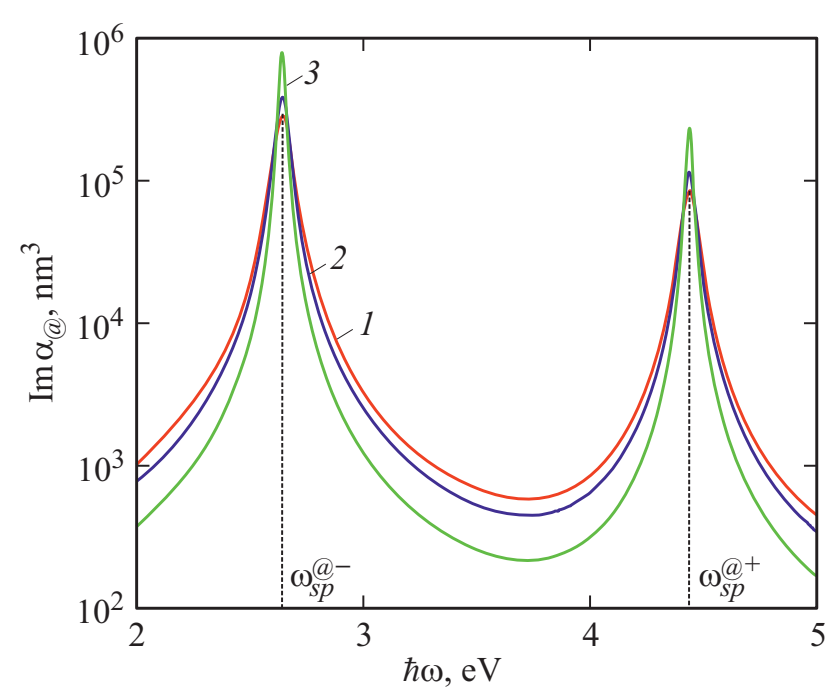

Рис. 2. Частотная зависимость мнимой части поляризуемости составной частицы $\mathrm{Au} @ \mathrm{Ag}\left(R_{\mathrm{c}}=20 \mathrm{~nm}, t=5 \mathrm{~nm}\right)$ в тефлоне

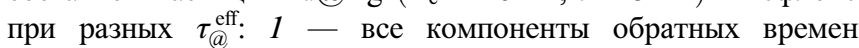
в $(11) \neq 0,2-$ компонента излучения $=0,3-$ компонента рассеяния $=0$.

$\left(R_{\mathrm{c}}=20 \mathrm{~nm}, t=5 \mathrm{~nm}, \quad\right.$ т. е. при выполнении условия $(10))$ в тефлоне при разных $\tau_{@}^{\mathrm{eff}} ; \omega_{s p}^{@-}$ и $\omega_{s p}^{@+}-$ означают пазмонные частоты первого и второго резонансов. Результаты расчетов свидетельствуют о необходимости учета всех каналов рассеяния, поскольку учет этих каналов по-отдельности приводит к завышенным значениям $\operatorname{Im} \alpha_{@}$ на частотах поверхностных плазмонных резонансов и заниженным значениям в остальном частотном интервале.

Конечно, всегда будут сложности с выбором размеров частицы для выполнения условий (9) и (10). Некоторые из траекторий $\left(\ell_{2}\right.$ на рис. 1$)$ не будут удовлетворять указанным границам применимости. Такое же замечание относится и к выводу компоненты (A9), особенно для крупных частиц (при условии $\ell_{\mathrm{c}}^{\text {bulk }} \ll 2 R_{\mathrm{c}}, \ell_{\mathrm{s}}^{\text {bulk }} \ll 2 t$ ). Тогда полученные результаты следует воспринимать как экстраполяционные, а влияние неточности вычислений компонент времени на основные характеристики можно оценить по рис. 2.

На рис. 3 приведены графики частотных зависимостей действительной и мнимой частей, а также модуля поляризуемости наносфер Au@Ag различных размеров. Отметим, что функция $\operatorname{Re} \alpha_{@}(\omega)$ является знакопере-

Используемые параметры, $a_{0}=\hbar^{2} /\left(m_{e} e^{2}\right)-$ боровский радиус

\begin{tabular}{c|c|c|c|c}
\hline Параметры & $\mathrm{Au}[20]$ & $\mathrm{Ag}[20]$ & $\mathrm{Pt}[33]$ & $\mathrm{Pd}[34]$ \\
\hline$r_{s}, a_{0}$ & 3.01 & 3.02 & 3.27 & 4.00 \\
$m^{\text {eff }} / m_{e}$ & 0.99 & 0.96 & 0.54 & 0.37 \\
$\epsilon^{\infty}$ & 9.84 & 3.70 & 4.42 & 2.52 \\
$\tau^{\text {bulk }}, 10^{-15} \mathrm{~s}$ & 29 & 40 & 9.5 & 7.2 \\
$\hbar \omega_{b p}, \mathrm{eV}$ & 9.07 & 9.17 & 10.9 & 9.70
\end{tabular}



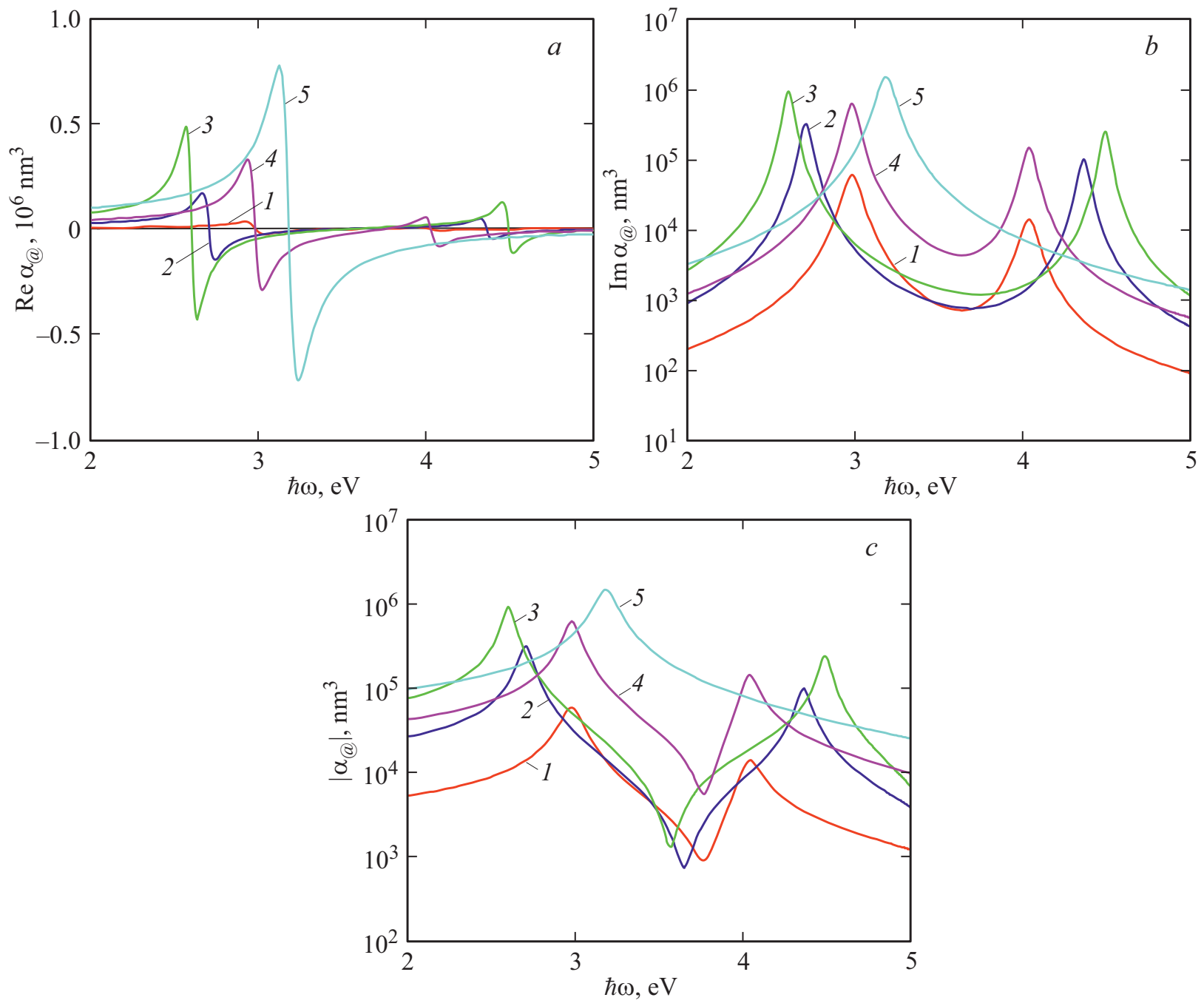

Рис. 3. Частотная зависимость дипольной поляризуемости сфер $\mathrm{Au} @ \mathrm{Ag}$ в тефлоне. Кривые $1-3$ при радиусе ядра $R_{\mathrm{c}}=v a r$ и толщине оболочки $t=5 \mathrm{~nm}$ (const): $1-R_{\mathrm{c}}=10 \mathrm{~nm}, 2-R_{\mathrm{c}}=20 \mathrm{~nm}, 3-R_{\mathrm{c}}=30 \mathrm{~nm}$. Кривые 4,5 при $R_{\mathrm{c}}=20 \mathrm{~nm}($ const) и толщине оболочки $t=v a r: 4-t=10 \mathrm{~nm} ; 5-t=20 \mathrm{~nm}$.

менной, в то время как $\operatorname{Im} \alpha_{@}(\omega)>0$ во всем диапазоне частот. Кроме того, увеличение радиуса ядра, т. е. увеличение фракции $\mathrm{Au}$ в наночастице (максимумы кривых в последовательности $1 \rightarrow 2 \rightarrow 3$ ), приводит к тому, что первые максимумы $\operatorname{Im} \alpha_{@}$ и $\left|\alpha_{@}\right|$ становятся более выраженными, а также к увеличению „расстояния“ $\left(\omega_{s p}^{@+}-\omega_{s p}^{@-}\right)$ или „отталкиванию“ между этими максимумами и увеличению их численного значения.

Из выражения для дипольной поляризуемости монометаллической сферы и условия поверхностного плазменного резонанса

$$
\operatorname{Re} \epsilon\left(\omega_{s p}\right)=-2 \epsilon_{\mathrm{m}}
$$

следует формула для частоты поверхностных плазмонов

$$
\omega_{s p}^{2}=\frac{\omega_{b p}^{2}}{\epsilon^{\infty}+2 \epsilon_{\mathrm{m}}}-\left(\frac{1}{\tau^{\text {bulk }}}+\mathcal{A} \frac{v_{\mathrm{F}}}{R}\right)^{2} .
$$

Из (17) можно ожидать очень слабый классический размерный красный сдвиг при уменьшении радиуса сферы [известно также, что от выбора модельного подхода возможны как синий [31], так и красный сдвиги поверхностного резонанса [32]].

Однако, в биметаллических сферах с уменьшением $R_{\mathrm{c}}$ при постоянной толщине оболочки $t$ (т.е. в целом радиус кластера $R$ уменьшается), как следует из рис. $3, b$, наблюдается синий сдвиг первого резонанса $\omega_{s p}^{@-}$ (максимумы кривых в последовательности $3 \rightarrow 2 \rightarrow 1$ сдвигаются вправо по шкале частот). Противоположный красный размерный сдвиг наблюдается для тех же кривых на втором резонансе $\omega_{s p}^{@+}$. Таким образом здесь, с уменьшением радиуса сферы резонансы $\omega_{s p}^{@-}$ и $\omega_{s p}^{@+}$ „притягиваются“ друг к другу.

Для первого максимума кривых рис. $3, b$ в последовательности $5 \rightarrow 4 \rightarrow 2$ ( $R_{\mathrm{c}}=$ const, $t$ уменьшается, и в целом $R$ уменьшается) наблюдается уже красный размерный сдвиг первого резонанса. В этом случае с уменьшением радиуса сферы резонансы $\omega_{s p}^{@-}$ и $\omega_{s p}^{@+}$ „отталкиваются“ друг от друга. 

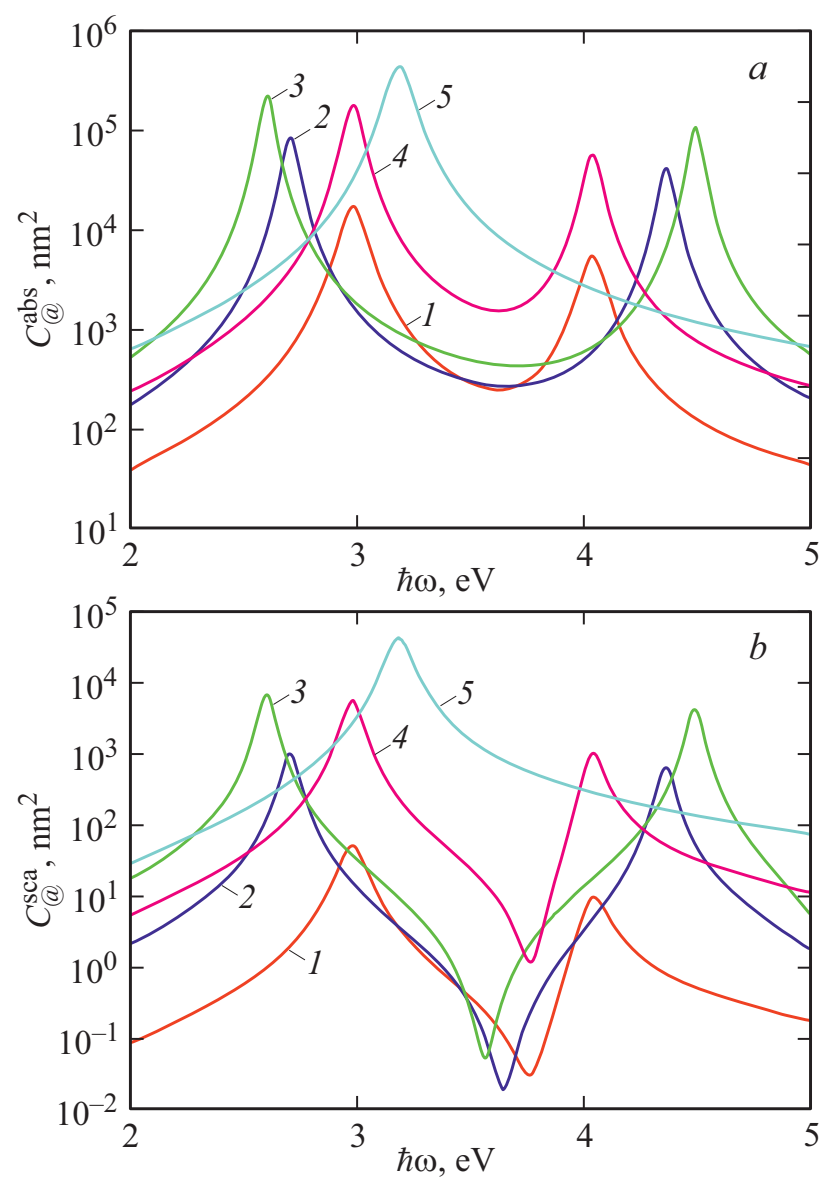

Рис. 4. Частотные зависимости сечений поглощения и рассеяния $\mathrm{Au} @ \mathrm{Ag}$ при тех же значениях параметров, что и на рис. 3.

Частотные зависимости сечений поглощения и рассеяния частиц $\mathrm{Au} @ \mathrm{Ag}$ приведены на рис. 4. Вследствие того, что $C_{@}^{\mathrm{abs}} \sim \operatorname{Im} \alpha_{@}$, характер кривых $C_{@}^{\mathrm{abs}}(\omega)$ подобен зависимостям $\operatorname{Im} \alpha_{@}(\omega)$. Кроме того, $C^{\mathrm{sca} @} \ll C_{@}^{\mathrm{abs}}$, поэтому $C_{@}^{\mathrm{ext}} \approx C_{@}^{\mathrm{abs}}$, и, таким образом, потери энергии падающей электромагнитной волны определяются преимущественно процессами поглощения.

На рис. 5 сравниваются частотные зависимости сечений поглощения биметаллических наночастиц одинакового размера, но разного элементного состава (Au@Pt, $\mathrm{Pt} @ \mathrm{Au}$ и Pt@Pd). Для всех трех систем заметны качественные и количественные различия кривых, но систематика кривых такая же как на рис. $3, b$. Так, перемена металлов ядра и оболочки (рис. 5, $a$ и $b$ ), во-первых, приводит к усилению размерного притяжения пиков $\mathrm{Au} @ \mathrm{Pt}$ на рис. 5, $a$ (синий/красный сдвиги) и пиков $\mathrm{Pt} @ \mathrm{Au}$ на рис.5, $b$ (красный/ слабый красный сдвиги), во-вторых, наблюдается заметный красный сдвиг всей картины резонансов на рис. $5, b$ по сравнению с рис. $5, a$, в основном связанный только с различием металлов, а не размерами. Как показано ниже, формально это объясняется разницей объемных плазмонных частоты $\left(\omega_{b p, \text { Аu }}<\omega_{b p, \text { Рt }}\right)$. Следует отметить, что разница между

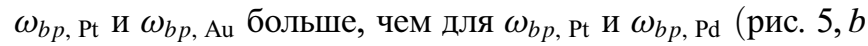
и $5, c)$, причем в последнем случае первые максимумы выражены сильнее вторых.

Соответствуют ли максимумы поляризуемости и сечений плазмонным резонансам ядра и оболочки?

В случае биметаллических частиц при анализе численных результатов приходится оперировать со значительным количеством параметров металлов (см. таблицу), и учитывать радиусы ядра и оболочки. Поэтому аналитическое рассмотрение весьма затруднительно при неизбежности прямых вычислений. Однако можно попытаться провести анализ в некоторых предельных случаях.

Напомним, что равенство нулю числителя и знаменателя в выражении (1) определяют, соответственно, условия невидимости и резонансной поляризуемости. Так, если поляризуемость частицы равна нулю, то она не дает вклад в рассеянное поле и не поглощает энергию внешней волны, поэтому сечения рассеяния и поглощения частицы равны нулю, а, следовательно, отсутствует рассеяние внешней волны. Этот эффект приводит к невозможности детектирования положения частицы, т. е. частица является невидимой. Условие равенства нулю действительной части знаменателя в (1) с учетом (4) приводит к уравнению относительно плазмонных частот $\omega_{s p}^{@ \pm}$.

Вследствие большого числа параметров задачи проведем анализ, начиная с приближения

$$
\tau_{@}^{\mathrm{eff}}=\infty
$$

которое означает отсутствие мнимой части у диэлектрической функций (4), т. е. нулевую ширину резонансов, а также равенство нулю скобки в (17). Условие (18) позволяет записать биквадратное уравнение относительно плазмонных частот $\omega_{s p}^{@}$

$$
a\left(\omega_{s p}^{@}\right)^{4}+b\left(\omega_{s p}^{@}\right)^{2}+c=0
$$

где

$$
\begin{gathered}
a=2\left(1-\beta_{\mathrm{c}}\right)\left(\epsilon_{\mathrm{s}}^{\infty}\right)^{2}+\left(1+\beta_{\mathrm{c}}\right) \epsilon_{\mathrm{c}}^{\infty} \epsilon_{\mathrm{s}}^{\infty} \\
+2\left(1-\beta_{\mathrm{c}}\right) \epsilon_{\mathrm{m}} \epsilon_{\mathrm{c}}^{\infty}+2\left(2+\beta_{\mathrm{c}}\right) \epsilon_{\mathrm{m}} \epsilon_{\mathrm{s}}^{\infty}, \\
b=\left(2 \epsilon_{\mathrm{m}}+\epsilon_{\mathrm{s}}^{\infty}-2 \beta_{\mathrm{c}}\left(\epsilon_{\mathrm{s}}^{\infty}-\epsilon_{\mathrm{m}}\right)\right) \omega_{b p, \mathrm{c}}^{2} \\
-\left(\epsilon_{\mathrm{c}}^{\infty}+4 \epsilon_{\mathrm{s}}^{\infty}+4 \epsilon_{\mathrm{m}}+2 \beta_{\mathrm{c}}\left(\epsilon_{\mathrm{c}}^{\infty}-\epsilon_{\mathrm{m}}\right)\right) \omega_{b p, \mathrm{~s}}^{2}, \\
c=\omega_{b p, \mathrm{~s}}^{2}\left[\left(1-2 \beta_{\mathrm{c}}\right) \omega_{b p, \mathrm{c}}^{2}+2\left(1-2 \beta_{\mathrm{c}}\right) \omega_{b p, \mathrm{~s}}^{2}\right] .
\end{gathered}
$$

Однако решение уравнения (19) в таком виде все еще остается слишком громоздким и требуются дальнейшие упрощения. С этой целью положим

$$
\epsilon^{\infty}, m^{\mathrm{eff}} / m_{e}=1, \quad \text { а также } \epsilon_{\mathrm{m}}=1
$$

(сфера находится в вакууме). Использование условий (18) и (21) упрощает уравнение (19) (что отражается на обозначениях $\left.\omega_{s p}^{* @}\right)$ и теперь его решение 

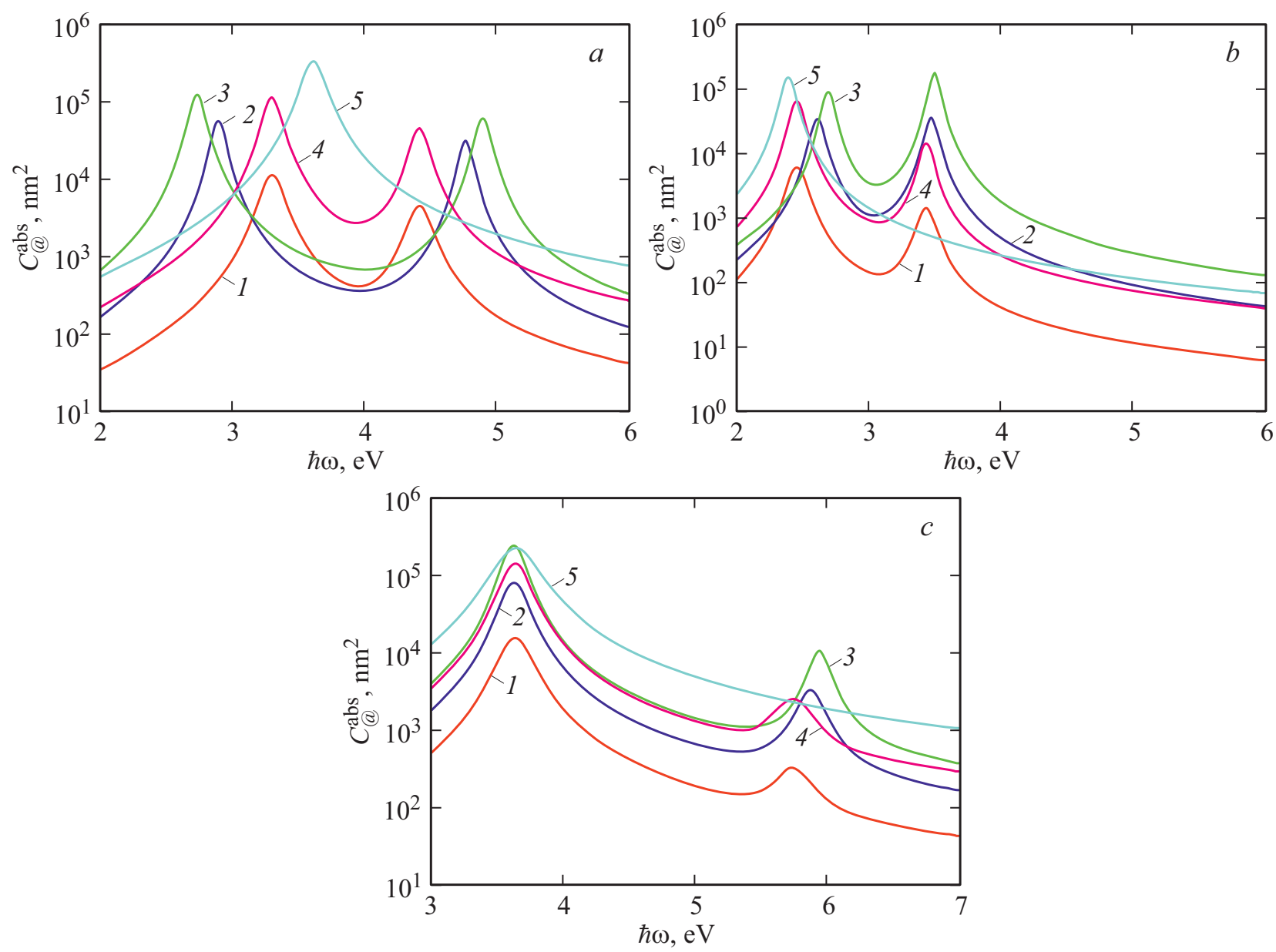

Рис. 5. Частотные зависимости сечения поглощения частиц $\mathrm{Au} @ \mathrm{Pt}(a), \mathrm{Pt} @ \mathrm{Au}(b)$ и $\mathrm{Pt} @ \mathrm{Pd}(c)$ при тех же значениях параметров, что и на рис. 3.

становится более наглядным

$$
\begin{aligned}
\left(\omega_{s p}^{* @ \pm}\right)^{2}= & \frac{1}{6}\left[\omega_{b p, \mathrm{c}}^{2}+3 \omega_{b p, \mathrm{~s}}^{2} \pm\left[\left(\omega_{b p, \mathrm{c}}^{2}+\omega_{b p, \mathrm{~s}}^{2}\right)^{2}\right.\right. \\
& \left.\left.-8 \beta_{\mathrm{c}} \omega_{b p, \mathrm{~s}}^{2}\left(\omega_{b p, \mathrm{c}}^{2}-\omega_{b p, \mathrm{~s}}^{2}\right)\right]^{1 / 2}\right] .
\end{aligned}
$$

Из него сразу следует наличие двух плазменных резонансов у биметаллической сферы.

Интересно отметить два предельных перехода в (22), превращающих биметаллическую частицу в две монометаллические, состоящие из разных металлов

$$
\begin{gathered}
\omega_{s p}^{* @-} \rightarrow \frac{\omega_{b p, \mathrm{~s}}}{\sqrt{3}} \equiv \omega_{s p, \mathrm{~s}} \quad \text { при } \quad \beta_{\mathrm{c}} \rightarrow 0 ; \\
\omega_{s p}^{* @+} \rightarrow \omega_{b p, \mathrm{c}} \quad \text { при } \quad \beta_{\mathrm{c}} \rightarrow 1 .
\end{gathered}
$$

Первый предел (23) (ядро исчезает) приводит к равенству частоты первого резонанса $\omega_{s p}^{* @-}$ частоте локализованного поверхностного плазмона оболочки $\omega_{s p, \mathrm{~s}}$.

Второй из этих переходов (24) (металл ядра заполняет весь объем, а оболочка исчезает) приводит к равенству частот поверхностного плазмона $\omega_{s p}^{* @+}$ и объемного плазмона ядра $\omega_{b p, c}$. Кажущееся парадоксальным исчезновение поверхностного плазмона на самом деле является следствием граничных условий, отраженных в формуле (1) для поверхности ядра, граничащего только с металлом оболочки, а не с внешней поверхностью.

В литературе популярной является идея использования, предложенной в [35], интуитивной схемы вычисления частот поверхностных плазмонов в слоистой сферической матрешке посредством комбинации уже известных результатов для частот плазмонов поверхностей положительной и отрицательной кривизны (шарика и полости). Формула (1) и предельный переход (24) указывают на принципиальную невозможность такого приема для частиц, ядро и оболочка которых являются металлическими.

Удобно использовать выражения (22) для определения знака производных

$$
\begin{aligned}
& \left.\frac{\partial \omega_{s p}^{* @ \pm}}{\partial \beta_{\mathrm{c}}} \frac{d \beta_{\mathrm{c}}}{d R_{\mathrm{c}}}\right|_{R=\mathrm{const}}=\mp \frac{\beta_{\mathrm{c}}}{R_{\mathrm{c}}} \Omega, \\
& \left.\frac{\partial \omega_{s p}^{* @ \pm}}{\partial \beta_{\mathrm{c}}} \frac{d \beta_{\mathrm{c}}}{d R}\right|_{R_{\mathrm{c}}=\mathrm{const}}= \pm \frac{\beta_{\mathrm{c}}}{R} \Omega,
\end{aligned}
$$


где

$$
\Omega=\frac{\left(\omega_{b p, \mathrm{c}}^{2}-\omega_{b p, \mathrm{~s}}^{2}\right)\left(\omega_{b p, \mathrm{~s}}^{2} / \omega_{s p}^{* @ \pm}\right)}{\left[\left(\omega_{b p, \mathrm{c}}^{2}+\omega_{b p, \mathrm{~s}}^{2}\right)^{2}-8 \beta_{\mathrm{c}} \omega_{b p, \mathrm{~s}}^{2}\left(\omega_{b p, \mathrm{c}}^{2}-\omega_{b p, \mathrm{~s}}^{2}\right)\right]^{1 / 2}} .
$$

Эти знаки и соответствуют размерным красным и синим сдвигам резонансов, упомянутым выше. Знаки производных управляются знаком скобки в числителе выражения (26), в которой присутствует разница квадратов частот объемных плазмонов разных металлов (см. таблицу). Например, при изменении архитектуры частицы А@В на „обратную“ структуру В@А, знак $\Omega(26)$ в (25) меняется на противоположный. На примеpe частиц $\mathrm{Ag} @ \mathrm{Au}$ и $\mathrm{Au} @ \mathrm{Ag}$ такой упрощенный анализ подтверждается прямыми вычислениями. Однако для комбинаций других металлов, когда важным является учет констант $\epsilon^{\infty}, m^{\text {eff }}$ и $\epsilon_{\mathrm{m}}$, такие упрощенные представления, по-видимому, оказываются недостаточными [при смене архитектуры $\mathrm{Au@Pt} \mathrm{(рис.} \mathrm{5,a)} \mathrm{на} \mathrm{Pt@Au}$ (рис. $5, b$ ) размерный сдвиг второго резонанса значительно ослабел, но все же остался красным].

Тем не менее, такое простое описание биметаллической сферы и, соответствующая ему формула (22), указывает, во-первых, на наличие двух резонансов по числу металлов в частице, что соответствует экспериментам (рис. 4 в [17] и рис. 4, $a$ в [22]), а, вовторых, позволяет выделить основные параметры задачи:

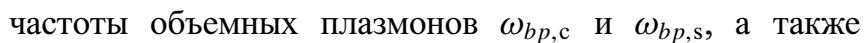
объемную фракцию ядра $\beta_{\mathrm{c}}$ [формула $(17)$ в этом приближении вообще не содержит размерной зависимости]. „Смешение б объемных плазмонов в соответствии с формулой (1) осуществляется посредством параметра $\beta_{\mathrm{c}}$. Для трехметаллической сферы, соответственно будет три плазменных резонансных пика. Для анализа такой ситуации пригодна формула для поляризуемости частицы с диэлектрическим ядром и двумя металлическими оболочками из работы [36], что является аналогом формулы (1) для двухслойной сферы. В случае сплавов, по-видимому, будет один резонанс.

Что касается прямых вычислений характеристик в более простом варианте (21), но с учетом (А3)-(А9), например, для $\mathrm{Au} @ \mathrm{Ag}$ и $\mathrm{Ag} @ \mathrm{Au}$, то они привели к смещению всей картины резонансов целиком примерно на удвоенные частоты с сохранением размерных сдвигов.

Полученные результаты неплохо согласуются с экспериментами, в которых, как правило, анализируется первый по частоте резонанс $\omega_{s p}^{@-}:$ в работе [17] на рис. 2, А, $\mathrm{B}$ для частиц $\mathrm{Ag} @ \mathrm{Au}$ виден сильный голубой размерный сдвиг при постоянном размере ядра $\mathrm{Ag}$ и уменьшении толщины оболочки $\mathrm{Au}$; аналогичная картина, но гораздо слабее, имеет место на рис. 4, $a$ в [22] для частиц $\mathrm{Au} @ \mathrm{Ag}$. Наши вычисления полностью согласуются с результатами [17], но в сравнении с [22] дают не слабый голубой, а красный сдвиг пика поглощения.

На рис. $6, a$ и $b$ в диапазоне $\hbar \omega=(1,15) \mathrm{eV}$ приведена рассчитанная частотная дисперсия радиационной эффективности крупных частиц разного состава и размеров, расположенных в тефлоне. Оба рисунка демонстрируют сильную зависимость от сорта металла и размеров ядра и оболочки. В видимой области спектра $(\hbar \omega \approx 1 \mathrm{eV})$ величина $\xi_{@}^{\mathrm{rad}}$ невелика, а на резонансных частотах, вследствие сильного поглощения $\left[C_{@}^{\mathrm{abs}}\left(\omega_{s p}^{@ \pm}\right) \gg C_{@}^{\mathrm{sca}}\left(\omega_{s p}^{@ \pm}\right)\right]$, имеет порядок $10^{-4}$. Близость величины $\xi_{@}^{\mathrm{rad}}$ к 1 наблюдается в средней и дальней ультрафиолетовой области, когда частицы обладают хорошими зеркальными свойствами. Такое же поведение при высоких частотах характерно и для частиц примерно вдвое или втрое меньших размеров. Однако в видимом диапазоне величина $\xi_{@}^{\mathrm{rad}}$ для них уменьшается более, чем на порядок.

В литературе имеются сведения, что в случае, когда металлические наночастицы размещены в полупроводнике со сложными зонами, то за счет плазмонных резонансов посредством ближнего поля можно добиться обратного эффекта - усиления взаимодействия с полупроводником, увеличивая тем самым эффективное сечение рассеяния и, соответственно, $\xi_{@}^{\mathrm{rad}}$.
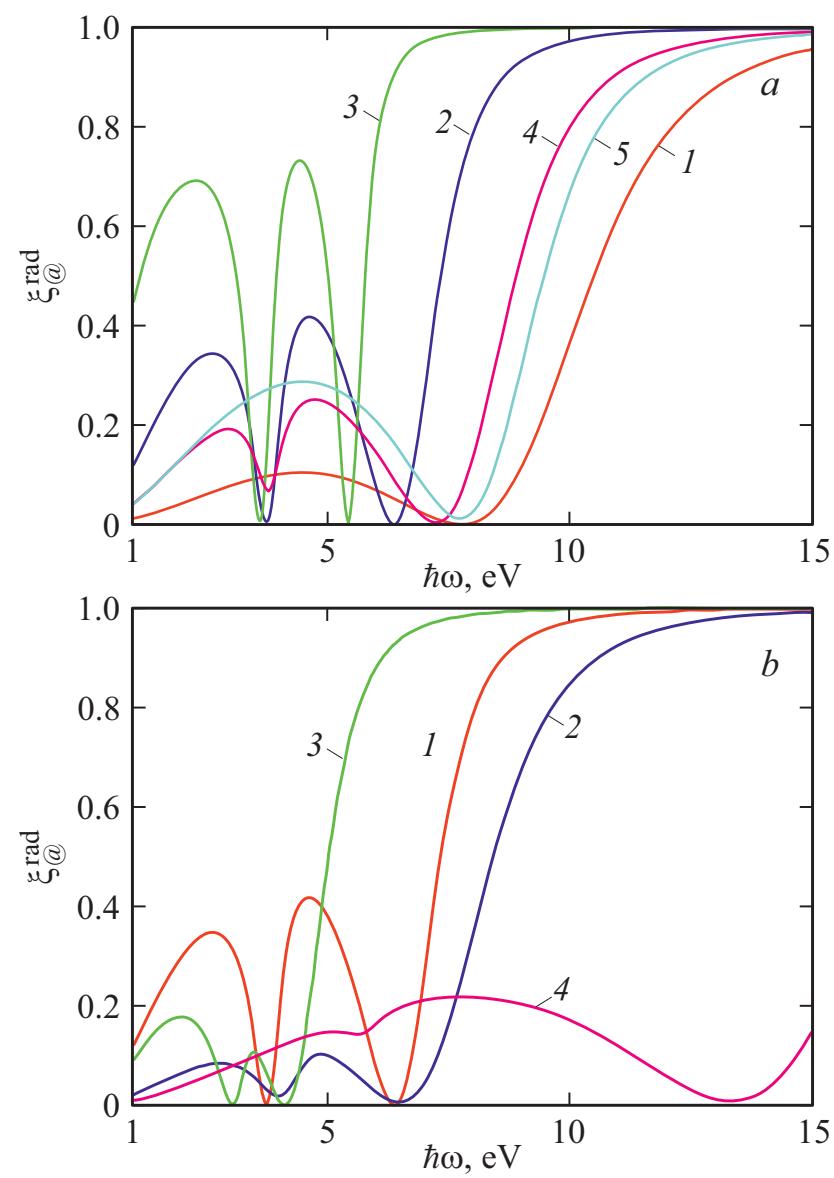

Рис. 6. Частотная зависимость радиационной эффективности для частицы $\mathrm{Au} @ \mathrm{Ag}(a): 1-R_{\mathrm{c}}=20 \mathrm{~nm}$ и $t=20 \mathrm{~nm}$, $2-R_{\mathrm{c}}=50 \mathrm{~nm}$ и $t=20 \mathrm{~nm}, 3-R_{\mathrm{c}}=100 \mathrm{~nm}$ и $t=20 \mathrm{~nm}$, $4-R_{\mathrm{c}}=50 \mathrm{~nm}$ и $t=30 \mathrm{~nm}, 5-R_{\mathrm{c}}=50 \mathrm{~nm}$ и $t=50 \mathrm{~nm}$; для разных частиц $(b): 1-\mathrm{Au} @ \mathrm{Ag}, 2-\mathrm{Au} @ \mathrm{Pt}, 3-\mathrm{Pt} @ \mathrm{Au}$, $4-\mathrm{Pt} @ \mathrm{Pd}$ с общими для них значениями $R_{\mathrm{c}}=50 \mathrm{~nm}$ и $t=20 \mathrm{~nm}$. 
Все эти особенности позволяют эффективно приспосабливать современные солнечные элементы к спектру излучения Солнца, в зависимости от географического положения на поверхности планеты или в космосе.

Еще одним практически важным применением наноплазмоники является фототермическая терапия злокачественных опухолей. После внешней подкачки света средняя температура сферы повышается на $\Delta T$. Если оптимальная температура сферы должна быть $42^{\circ} \mathrm{C}$, то $\Delta T=42-36.6=5.4^{\circ}$.

Повышение температури частицы, вследствие поглощения внешнего поля, можно оценить как

$$
\Delta T \approx \frac{I_{0}}{4 \pi R \kappa} C_{@}^{\mathrm{abs}},
$$

где $I_{0}$ - интенсивность падающего света; $\kappa-$ теплопроводность окружающей среды [37].

Оценки проведем для двух частиц $\mathrm{Au} @ \mathrm{Ag} / \mathrm{Ag} @ \mathrm{Au}$ c параметрами $R_{c}=10 \mathrm{~nm}$ и $t=5 \mathrm{~nm}, \kappa=0.25 \mathrm{~W} / \mathrm{m} \cdot \mathrm{K}$ (тефлон), а также значением $I_{0}=1 \mathrm{~mW} / \mu \mathrm{m}^{2}$ для зеленого света $(\lambda=530 \mathrm{~nm})$ из работы [37]. Используя данные рис. 4, $a$ (кривая 1), при $\hbar \omega_{s p}^{\varrho--}=2.98 \mathrm{eV}$ для $\mathrm{Au} @ \mathrm{Ag}$, и заново вычисляя при $\hbar \omega_{s p}^{@-}=2.44 \mathrm{eV}$ для $\mathrm{Ag} @ \mathrm{Au}$, имеем $C_{@}^{\mathrm{abs}}=17.1 \cdot 10^{3} \mathrm{~nm}^{2} / 8.04 \cdot 10^{3} \mathrm{~nm}^{2}$, и $\Delta T \approx 360^{\circ} / 150^{\circ}$ для $\mathrm{Au} @ \mathrm{Ag} / \mathrm{Ag} @ \mathrm{Au}$, соответственно.

Если заменить тефлон на воду $(\kappa=0.6 \mathrm{~W} / \mathrm{m} \cdot \mathrm{K}$, $\left.\epsilon_{\mathrm{m}}=1.77\right)$, то $\Delta T \approx 150^{\circ} / 60^{\circ}$. Такой нагрев приблизительно на порядок превышает необходимый, но может быть легко скорректирован путем замены материала, изменения параметров сферы, а также расстояния $r$ от нее, поскольку пространственное распределение температуры при $r>R$ выглядит как $\Delta T / r$.

Если на практике удается организовать конгломерат близко расположенных наносфер, как в [17] и [22], это позволяет увеличить на порядки величин поле внутри конгломерата. Величина такого усиления характеризуется фактором

$$
\mathscr{G}(r)=\left|1+2 \alpha / r^{3}\right|^{2},
$$

где $r>R$ - расстояние от центра сферы до точки, для которой рассчитывается фактор усиления поля [38]. Эта характеристика легко вычисляется в нашей схеме. Тогда, можно утверждать, что это усиление позволит уменьшить величину $I_{0}$ в формуле (27) на порядки величины для того, чтобы достичь нужной температуры при терапии злокачественных опухолей. Возможно даже, что такая терапия будет происходить „сама по себе“, т.е. вовсе без фиксированного внешнего источника излучения $\left(I_{0}=0\right)$, а лишь за счет слабых случайных источников, на длинах волн, которые соответствуют окну прозрачности биологической ткани.

Отметим, что результаты представленной работы получены в предположении однородности поля внутри частицы, т. е. при выполнении условия $\xi \ll 1$ [см. (14)]. Такое неравенство хорошо выполняется в далекой инфракрасной области и вблизи плазменного резонанса, когда $\epsilon_{@}(\omega) \rightarrow 0$ и скин-эффектом можно пренебречь.
На границах исследуемого диапазона частот и максимальных размеров наших частиц параметр $\xi \leq 1$.

Скин-эффект в сферах, тонких металлических пластинах и проволоках рассматривался неоднократно (см., например [31,32,39-42]). Для этого применялся как гидродинамический, так и кинетический подходы. Последний использовался для частиц при $\zeta \ll 1[25,27]$.

Оценка глубины скин-слоя для полубесконечного $\mathrm{Au}$ при $\hbar \omega=1 \mathrm{eV}$ дает величину в $5 \mathrm{~nm}$, что фактически совпадает с толщиной нашей оболочки $t$ при максимальном внешнем радиусе $50 \mathrm{~nm}$. Параметр зеркальности поверхности для тонких образцов можно считать равным нулю $[40,42]$.

Специфика нашей постановки задачи заключается в том, что для всех каналов рассеяния длина свободного пробега фактически определяется внешним радиусом частицы. Таким образом, невозможно классифицировать к нормальному или аномальному скин-эффекту относится поглощение электромагнитной волны. Это находит подтверждение и в диаграмме на рис. 1 работы [41]. Наши параметры модели попадают прямо в центр „площади четырех углов“" на этой диаграмме. Возможно режим поглощения относится к специфическому „квазинормальному“ скин-эффекту, отмеченному в [39] для тонкой пластинки, но оценить его для сферической симметрии не представляется возможным.

В связи с вышесказанным, авторы предлагают рассматривать результаты данной работы на границах исследуемого диапазона частот и максимальных размеров частиц как оценочные.

\section{4. Заключение}

В приближении диффузного рассеяния получены выражения для поверхностной, радиационной и объемной компонент эффективного времени релаксации электронов в случаях, когда длина свободного пробега электронов меньше или несколько больше характерных размеров ядра и оболочки биметаллической наночастицы. Показано, что учет всех компонент времени релаксации необходим для точного определения мнимой части поляризуемости, а соответственно и величины поглощения, на частоте поверхностного плазмонного резонанса.

Компоненты эффективного времени релаксации являются своего рода „перекрестными“ и создают эффект „внутреннего перемешивания“ диэлектрических функций ядра и оболочки в нашей задаче, как, например, это происходит во „внешнем перемешивании“ диэлектрических функций согласно электродинамической формуле для общей диэлектрической функции биметаллической сферы в диэлектрике. Эти компоненты невозможно целиком отнести либо к ядру, либо к оболочке.

Проведены расчеты частотных зависимостей поляризуемости, сечений поглощения и рассеяния, а также радиационной эффективности для наночастиц различного элементного состава в тефлоне, с различными значениями радиусов ядра и толщины оболочек. Результаты 
демонстрируют возможность управления характеристиками поглощения и рассеяния внешней волны. Так, при одинаковых частотах вблизи резонансов величина сечения рассеяния оказывается значительно меньше, чем сечения поглощения, поэтому можна считать, что потери энергии падающей электромагнитной волны в соответствующем композите определяются преимущественно процессами поглощения.

С помощью упрощенной теории Друде выявлено, что наличие двух резонансов соответствует только числу металлов в частице, а основными параметрами задачи являются частоты объемных плазмонов и объемные фракции этих металлов. „Смешение“ объемных плазмонов осуществляется посредством параметра объемной фракции материала ядра. В этом же смысле нельзя точно соотнести оба плазменных резонанса к конкретному металлу.

Например, для частицы Au@Pt с уменьшением радиуса ядра при постоянной толщине оболочки, а также, когда радиус ядра неизменный, а толщина оболочки уменьшается (т.е. в целом радиус частицы уменьшается) наблюдается заметный синий сдвиг первого и красный сдвиг второго резонансов. Для частицы с обратной архитектурой $\mathrm{Pt} @ \mathrm{Au}$ при тех же манипуляциях с размерами наблюдается уже красный сдвиг обоих резонансов. В зависимости от параметров металлов при уменьшении радиуса сферы резонансы могут как „притягиваться“, так и „отталкиваться“ друг от друга. Тем самым показана принципиальная невозможность применения схемы „гибридизации“ [35] для вычисления частот поверхностных плазмонов в слоистой сферической матрешке посредством комбинации уже известных результатов для частот плазмонов поверхностей положительной и отрицательной кривизны (шарика и полости).

Характер кривых частотных зависимостей сечения поглощения и радиационной эффективности существенно зависит от элементного состава и морфологии наночастицы. Так, инверсия металлов ядра и оболочки может приводить как к „красному“, так и „синему“ сдвигам экстремумов сечений поглощения и радиационной эффективности. Рассчитанная частотная дисперсия радиационной эффективности крупных частиц разного состава и размеров, расположенных в тефлоне, близка к единице в средней и дальней ультрафиолетовой области, когда частицы обладают хорошими зеркальными свойствами.

Оценено повышение температуры вблизи биметаллических сфер в различных средах вследствие поглощения электромагнитной волны от внешних источников. В качестве ремарки можно отметить возможность слабого нагревания сфер в радиочастотном диапазоне, выгодном для глубинной терапии злокачественных новообразований.

\section{Благодарности}

Авторы благодарят В.П. Курбацкого за обсуждение результатов работы.

\section{Конфликт интересов}

У авторов нет конфликта интересов.

\section{Приложение А: Расчет времен релаксации}

Поверхностное рассеяние. Для благородных металлов при комнатной температуре $\left(\ell^{\text {bulk }} \simeq 10-100 \mathrm{~nm}\right.$, длина волны де Бройля электронов $\sim 0.3 \mathrm{~nm})$ существенным оказываются неровности поверхности атомного масштаба. В связи с этим мы предполагаем, что рассеяние электронов на поверхности биметаллической сферической частицы является диффузным. Условием применения классического подхода к описанию рассеяния является неравенство $\hbar \omega \ll \varepsilon_{\mathrm{F}}$.

Выражение для вероятности рассеяния электронов по всем направлениям, под углами, которые лежат в интервале между $(\theta, \theta+d \theta)$ и $(\varphi, \varphi+d \varphi)$ по отношению к касательной плоскости сферы (рис. 1), имеет вид

$$
d W=W_{0} \cos \theta d \theta d \varphi,
$$

$\theta \in(0, \pi / 2), \varphi \in(0,2 \pi)$.

Фактически речь идет об усреднении вектора (длины свободного пробега) $\vec{\ell}$ по всем направлениям. Для „докритических“ углов $\theta \leq \theta^{*}$, где $\cos \theta^{*}=R_{\mathrm{c}} / R$, электрон рассеивается в металле оболочки, а для $\theta>\theta^{*}$ рассеивается в обоих металлах. В этом случае запишем $\vec{\ell}$ в виде суммы коллинеарных векторов $\vec{\ell}=\vec{\ell}_{1}+\vec{\ell}_{2}+\vec{\ell}_{3}$ и среднее суммы будет равно сумме средних.

При преобразовании вектора в следующий вид $\vec{\ell}_{1} / v_{\mathrm{F}, \mathrm{s}}+\vec{\ell}_{2} / v_{\mathrm{F}, \mathrm{c}}+\vec{\ell}_{3} / v_{\mathrm{F}, \mathrm{s}}$ его направление не изменится (рассеянием на границе металлов пренебрегаем), а изменится только длина (теперь уже размерности времени), поэтому усреднение по направлениям проводится точно так же.

Среднее время релаксации на поверхности определяется как

$$
\left\langle\tau_{@}^{\text {surf }}\right\rangle_{R}=\frac{\int \tau_{@}^{\text {surf }} d W}{\int d W} .
$$

Тогда

$$
\begin{aligned}
\left\langle\tau_{@}^{\text {surf }}\right\rangle_{R}= & \int_{0}^{\theta^{*}} \frac{2 R \sin \theta}{v_{\mathrm{F}, \mathrm{s}}} \cos \theta d \theta \\
& +\int_{\theta^{*}}^{\pi / 2}\left(\frac{\ell_{1}}{v_{\mathrm{F}, \mathrm{s}}}+\frac{\ell_{2}}{v_{\mathrm{F}, \mathrm{c}}}+\frac{\ell_{3}}{v_{\mathrm{F}, \mathrm{s}}}\right) \cos \theta d \theta .
\end{aligned}
$$

Решая геометрическую задачу, имеем квадратное уравнение $\ell_{1}^{2}=R_{\mathrm{c}}^{2}-R^{2}+2 \ell_{1} R \sin \theta$, решением которого является

$$
\ell_{1}=R \sin \theta-\left(R_{\mathrm{c}}^{2}-R^{2} \cos ^{2} \theta\right)^{1 / 2}
$$

а также

$$
\ell_{2}=\left(R^{2}-R_{\mathrm{c}}^{2}-\ell_{1}^{2}\right) / \ell_{1}, \quad \ell_{3}=2 R \sin \theta-\ell_{2}-\ell_{1} .
$$


Предельные переходы, превращающие биметаллическую частицу в две разные монометаллические частицы, проще сделать на этом этапе.

Полагая $R_{\mathrm{c}}, \beta_{\mathrm{c}} \rightarrow 0, \quad$ имеем $\quad$ в $\quad(\mathrm{A} 2) \quad \theta^{*} \rightarrow \pi / 2$, $\ell_{2} / v_{\mathrm{F}, \mathrm{c}} \rightarrow 0$ и в результате

$$
\left\langle\tau_{@}^{\mathrm{surf}}\right\rangle_{R} \rightarrow R / v_{\mathrm{F}, \mathrm{s}}
$$

Полагая $R_{\mathrm{c}} \rightarrow R, \beta_{\mathrm{c}} \rightarrow 1, \quad$ имеем $\quad$ в $(\mathrm{A} 2) \quad \theta^{*} \rightarrow 0$, $\left(\ell_{1}+\ell_{3}\right) / v_{\mathrm{F}, \mathrm{s}} \rightarrow 0$ и в результате

$$
\left\langle\tau_{@}^{\mathrm{surf}}\right\rangle_{R} \rightarrow R / v_{\mathrm{F}, \mathrm{c}}
$$

Рассчитывая интегралы в (А2) с учетом выражений для $\ell_{1}, \ell_{2}$ и $\ell_{3}$, окончательно получаем

$$
\begin{gathered}
\left\langle\tau_{@}^{\text {surf }}\right\rangle_{R}=\frac{R}{\mathscr{A} v_{\mathrm{F}, \mathrm{s}}} \\
\frac{1}{\mathscr{A}}=1+\left(\frac{v_{\mathrm{F}, \mathrm{s}}}{v_{\mathrm{F}, \mathrm{c}}}-1\right) \\
\times\left[\beta_{\mathrm{c}}^{1 / 3}+\frac{1}{2}\left(1-\beta_{\mathrm{c}}^{2 / 3}\right) \ln \left(\frac{1-\beta_{\mathrm{c}}^{1 / 3}}{1+\beta_{\mathrm{c}}^{1 / 3}}\right)\right] .
\end{gathered}
$$

Радиационное затухание. Используя в формуле (8)

$$
\operatorname{Re} \sigma(\omega)=\frac{\sigma_{0}}{1+\left(\omega \tau^{\text {eff }}\right)^{2}}, \quad \sigma_{0}=\frac{e^{2} \bar{n} \tau^{\text {eff }}}{m^{\text {eff }}},
$$

a также неравенство в области плазменных частот $\omega \tau^{\mathrm{eff}} \gg 1$ и, следовательно, замену $\omega \rightarrow \omega_{b p}$, получаем

$$
\left\langle\tau_{@}^{\mathrm{rad}}\right\rangle_{R}=\frac{9}{2} c^{3} \epsilon_{\mathrm{m}}^{1 / 2}\left\langle\mathscr{B} \tau^{\mathrm{rad}}\right\rangle_{R}, \quad \mathscr{B}=\frac{1}{\omega_{b p}^{3} V} .
$$

1. Случай (9). Аналогично (А2) запишем

$$
\begin{aligned}
\left\langle\tau_{@}^{\mathrm{rad}}\right\rangle_{R} & =\frac{9}{2} c^{3} \epsilon_{\mathrm{m}}^{1 / 2}\left[\int_{0}^{\theta^{*}} \mathscr{B}_{\mathrm{s}} \frac{2 R \sin \theta}{v_{\mathrm{F}, \mathrm{s}}} \cos \theta d \theta\right. \\
& \left.+\int_{\theta^{*}}^{\pi / 2}\left(\mathscr{B}_{\mathrm{s}} \frac{l_{1}}{v_{\mathrm{F}, \mathrm{s}}}+\mathscr{B}_{\mathrm{c}} \frac{l_{2}}{v_{\mathrm{F}, \mathrm{c}}}+\mathscr{B}_{\mathrm{s}} \frac{l_{3}}{v_{\mathrm{F}, \mathrm{s}}}\right) \cos \theta d \theta\right] .
\end{aligned}
$$

Коэффициент $\mathscr{B}_{\mathrm{c}}$ содержит объем ядра $V_{\mathrm{c}}=4 \pi R_{\mathrm{c}}^{3} / 3$ $=\beta_{\mathrm{c}} V_{0}, \quad$ а коэффициент $\mathscr{B}_{\mathrm{s}}-$ объем оболочки $V_{\mathrm{s}}=4 \pi\left(R^{3}-R_{\mathrm{c}}^{3}\right) / 3=\left(1-\beta_{\mathrm{c}}\right) V_{0}$, где $V_{0}=4 \pi R^{3} / 3-$ полный объем.

2. Случай (10). Тогда

$$
\begin{aligned}
\left\langle\tau_{@}^{\mathrm{rad}}\right\rangle_{R}= & \frac{9}{2} c^{3} \epsilon_{\mathrm{m}}^{1 / 2}\left[\int_{0}^{\theta^{*}} \mathscr{B}_{\mathrm{s}} \tau_{\mathrm{s}}^{\text {bulk }} \cos \theta d \theta\right. \\
& \left.+\int_{\theta^{*}}^{\pi / 2}\left(2 \mathscr{B}_{\mathrm{s}} \tau_{\mathrm{s}}^{\text {bulk }}+\mathscr{B}_{\mathrm{c}} \tau_{\mathrm{c}}^{\text {bulk }}\right) \cos \theta d \theta\right] .
\end{aligned}
$$

В результате интегрирования в (А5) и (А6) получаем, соответственно, выражения

$$
\begin{aligned}
& \left\langle\tau_{@}^{\mathrm{rad}}\right\rangle_{R}=\frac{9}{2} \frac{\epsilon_{\mathrm{m}}^{1 / 2}}{V_{0}} \frac{R}{v_{\mathrm{F}, \mathrm{s}}} \frac{c^{3}}{\omega_{b p, \mathrm{~s}}^{3}} \\
& \times\left[\frac{1-\beta_{\mathrm{c}}^{1 / 3}-\frac{1}{2}\left(1-\beta_{\mathrm{c}}^{2 / 3}\right) \ln \frac{1-\beta_{\mathrm{c}}^{1 / 3}}{1+\beta_{\mathrm{c}}^{1 / 3}}}{1-\beta_{\mathrm{c}}}\right. \\
& \left.+\frac{v_{\mathrm{F}, \mathrm{s}}}{v_{\mathrm{F}, \mathrm{c}}} \frac{\omega_{b p, \mathrm{~s}}^{3}}{\omega_{b p, \mathrm{c}}^{3}} \frac{\beta_{\mathrm{c}}^{1 / 3}+\frac{1}{2}\left(1-\beta_{\mathrm{c}}^{2 / 3}\right) \ln \frac{1-\beta_{\mathrm{c}}^{1 / 3}}{1+\beta_{\mathrm{c}}^{1 / 3}}}{\beta_{\mathrm{c}}}\right],
\end{aligned}
$$

и

$$
\begin{aligned}
\left\langle\tau_{@}^{\mathrm{rad}}\right\rangle_{R}= & \frac{9}{2} \frac{\epsilon_{\mathrm{m}}^{1 / 2}}{V_{0}} \frac{c^{3}}{\omega_{b p, \mathrm{~s}}^{3}}\left[\frac{\left[2-\left(1-\beta_{\mathrm{c}}^{2 / 3}\right)^{1 / 2}\right] \tau_{\mathrm{s}}^{\text {bulk }}}{1-\beta_{\mathrm{c}}}\right. \\
& \left.+\frac{\omega_{b p, \mathrm{~s}}^{3}}{\omega_{b p, \mathrm{c}}^{3}} \frac{\left[1-\left(1-\beta_{\mathrm{c}}^{2 / 3}\right)^{1 / 2}\right] \tau_{\mathrm{c}}^{\text {bulk }}}{\beta_{\mathrm{c}}}\right] .
\end{aligned}
$$

Объемное рассеяние. Сохраняя стиль вычислений в диффузном приближении, запишем

$$
\begin{aligned}
\left\langle\tau_{@}^{\text {bulk }}\right\rangle_{R} & =\int_{0}^{\theta^{*}} \tau_{\mathrm{s}}^{\text {bulk }} \cos \theta d \theta+\int_{\theta^{*}}^{\pi / 2}\left(2 \tau_{\mathrm{s}}^{\text {bulk }}+\tau_{\mathrm{c}}^{\text {bulk }}\right) \cos \theta d \theta \\
& =\left[2-\left(1-\beta_{\mathrm{c}}^{2 / 3}\right)^{1 / 2}\right] \tau_{\mathrm{s}}^{\text {bulk }}+\left[1-\left(1-\beta_{\mathrm{c}}^{2 / 3}\right)^{1 / 2}\right] \tau_{\mathrm{c}}^{\text {bulk }} .
\end{aligned}
$$

Полагая $R_{\mathrm{c}}, \beta_{\mathrm{c}} \rightarrow 0$, следует положить $\tau_{\mathrm{c}}^{\text {bulk }} \rightarrow 0$. Тогда $\left\langle\tau_{@}^{\text {bulk }}\right\rangle_{R}=\tau_{\mathrm{s}}^{\text {bulk }}$.

Полагая $R_{\mathrm{c}}, \beta_{\mathrm{c}} \rightarrow 1$, следует положить $\tau_{\mathrm{s}}^{\text {bulk }} \rightarrow 0$. Тогда $\left\langle\tau_{@}^{\text {bulk }}\right\rangle_{R}=\tau_{\mathrm{c}}^{\text {bulk }}$.

Когда статья была написана, мы познакомились с работой [43], в которой рассмотрено классическое рассеяние электронов в двухслойной сферической частице и введен в формуле (37) критический угол (в нашей работе это $\theta^{*}$ на рис. 1 и в Приложении).

\section{Список литературы}

[1] S.A. Maier. Plasmonics: fundamentals and applications. Springer Science \& Business Media (2007).

[2] V. Amendola, R. Pilot, M. Frasconi, O.M. Maragò, M.A. Iatì. J. Phys.: Condens. Matter. 29, 203002 (2017).

[3] В.И. Балыкин, П.Н. Мелентьев. УФН 188, 143 (2018).

[4] А.В. Коротун, А.А. Коваль. Оптика и спектроскопия 127, 1032 (2019).

[5] W. Srituravanich, N. Fang, C. Sun, Q. Luo, X. Zhan. Nano Lett. 4, 1085 (2004).

[6] U. Schröter, A. Dereux. Phys. Rev. B 64, 125420 (2001).

[7] A. Ambrosio, B. Piccirillo, A. Sasso, E. Santamato. Opt. Commun. 230, 337 (2004).

[8] S. Nie, S.R. Emory. Science 275, 1102 (1997). 
[9] B. Špačková, P. Wrobel, M. Bocková, J. Homola. Proc. IEEE 104, 2380 (2016).

[10] H.A. Atwater, A. Polman. Nature Mater. 9, 205 (2010).

[11] В.А. Миличко, А.С. Шалин, И.С. Мухин, А.Э. Ковров, А.А. Красилин, А.В. Виноградов, П.А. Белов, К.Р. Симовский. УФН 186, 801 (2016).

[12] E.M. Larsson, C. Langhammer, I. Zorić, B. Kasemo. Science 326, 1091 (2009).

[13] D.J. De Aberasturi, A.B. Serrano-Montes, L.M. Liz-Marzán. Adv. Opt. Mater. 3, 602 (2015).

[14] F. Zarrinkhat, A.F. Najafabadi, T. Pakizeh. J. Opt. 19, 125003 (2017).

[15] C. Zhang, H. Zhao, L. Zhou, A.E. Schlather, L. Dong, M.J. McClain, D.F. Swearer, P. Nordlander, N.J. Halas. Nano Lett. 16, 6677 (2016).

[16] C.C. Wang, Y.C. Hsueh, C.Y. Su, C.C. Kei, T.P. Perng. Nanotechnol. 26, 254002 (2015).

[17] M.M. Moskovits, I. Srnová-Šloufová, B. Vlčková. J. Chem. Phys. 116, 10435 (2002).

[18] N. Valizade-Shahmirzadi, T. Pakizeh. Mater. Res. Express. 5, 04538 (2018).

[19] S. Sompech, S. Thaomola, A. Chingsungnoen, T. Dasri. Mater. Res. Express. 6, 026201 (2019).

[20] А.В. Коротун, А.А. Коваль, В.И. Рева, И.Н. Титов. ФММ 120, 1136 (2019).

[21] А.В. Скиданенко, Л.А. Авакян, Е.А. Козинкина, Л.А. Бугаев. ФТT 61, 115 (2019).

[22] Z. Gao, K. Deng, X. Wang, M. Miro, D. Tang. ACS Appl. Mater. Interfaces 6, 18243 (2014).

[23] Г. Хюлст. Рассеяние света малыми частицами. ИИЛ, М. (1961). $536 \mathrm{c}$.

[24] U. Kreibig, M. Vollmer. Optical properties of metal clusters. Springer, Berlin. (1995).

[25] N.I. Grigorchuk, P.M. Tomchuk. Phys. Rev. B 84, 085448 (2011).

[26] G.N. Blackman III, D.A. Genov. Phys. Rev. B 97, 115440 (2018).

[27] N.I. Grigorchuk. J. Opt. Soc. Am. B 29, 3404 (2012).

[28] В.П. Курбацкий, В.В. Погосов. Пис. ЖТФ 26, 84 (2000).

[29] Л.Д. Ландау, Е.М. Лифшиц. Электродинамика сплошных сред. Физматлит, М. (2001). 656 с.

[30] K. Tanabe. Mater. Lett. 61, 4573 (2007).

[31] R. Ruppin. J. Phys. Chem. Solids 39, 233 (1978).

[32] M. Cini. J. Opt. Soc. Am. 71, 386 (1981).

[33] M.A. Ordal, L.L. Long, R.J. Bell, S.E. Bell, R.R. Bell, R.W. Alexander, Jr., C.A. Ward. Appl. Opt. 22, 1099 (1983).

[34] W.E. Vargas. Appl. Opt. 56, 1266 (2017)

[35] E. Prodan, C. Radloff, N.J. Halas, P. Nordlander. Science 302, 419 (2003).

[36] Н.В. Селина. Рос. нанотехн. 14, 59 (2019).

[37] G. Baffou, R. Quidant, F.J. Garcia de Abajo. ACS Nano 4, 709 (2010).

[38] K. Tanabe. J. Phys. Chem. C 112, 15721 (2008).

[39] Б.М. Болотовский. ЖЭТФ 32, 559 (1957) [Sov. Phys. JЕTP 5, 465 (1957)].

[40] M.L. Theye. Phys. Lett. 25A, 764 (1967).

[41] G.R. Henry. J. Appl. Phys. 43, 2996 (1972).

[42] И.О. Моисеев, А.А. Юшканов, Ю.И. Яламов. ЖТФ 74, 87 (2004).

[43] A. Moroz. J. Phys. Chem. C 112, 10641 (2008).

Редактор Т.Н. Василевская 